\title{
Effects of Family Involvement in Nursing Mode on Postoperative Depression and Anxiety in Patients with Laryngeal Cancer
}

\author{
Jie Zhang ${ }^{1, *}{ }^{*}$, Yingxia Peng ${ }^{2, \dagger}$ \\ ${ }^{1}$ Department of First Class Ward, The First Affiliated Hospital, Jinan University, Guangzhou, China \\ ${ }^{2}$ Department for VIP, The First Affiliated Hospital, Jinan University, Guangzhou, China \\ Email address: \\ zhangjie@jnu.edu.cn (Jie Zhang) \\ ${ }^{*}$ Corresponding author \\ $\dagger$ Jie Zhang and Yingxia Peng are co-first authors.
}

\section{To cite this article:}

Jie Zhang, Yingxia Peng. Effects of Family Involvement in Nursing Mode on Postoperative Depression and Anxiety in Patients with Laryngeal Cancer. American Journal of Nursing Science. Vol. 9, No. 2, 2020, pp. 70-73. doi: 10.11648/j.ajns.20200902.16

Received: January 22, 2020; Accepted: February 26, 2020; Published: April 1, 2020

\begin{abstract}
Objective: The impact of family involvement in nursing models on depression and serious complications in patients with laryngeal cancer. Evaluate the effect of family intervention on nursing mode. Methods: The data of 40 patients with laryngeal cancer who undergo surgical treatment were collected and divided into observation group $(n=20)$ and budget $(n=20)$. In addition, the patients simply received conventional laryngeal cancer nursing measures, and the observation group patients based on family involvement in nursing mode measures. The depression and anxiety of the two groups were scored using the SAS scale and the SDS scale, and the quality of life was assessed using the quality of life assessment scale for statistical analysis. Results: There was no significant difference in SAS and SDS scores between admission and admission $(P<0.05)$. The SAS and SDS scores evaluated at discharge were significantly lower than those at admission $(P<0.05)$. The SAS and SDS scores of the observation group were significantly lower. All were significantly reduced (all $P<0.05)$. Group $(P<0.05)$. Patient satisfaction in the observation group also exceeded patient satisfaction. Conclusion: The family care mode can be implemented on the basis of routine nursing, which can improve the depression and anxiety of patients and improve their quality of life, which has certain clinical application value.
\end{abstract}

Keywords: Laryngeal Cancer, Anxiety, Depression, Quality of Life, Family Involvement in Nursing Model

\section{Introduction}

Laryngeal cancer is the most common malignant tumor of the head and neck. Its incidence accounts for $1.2 \%$ to $1.6 \%$ of systemic malignant tumors and $3.3 \%$ to $8.1 \%$ of malignant tumors of the head and neck [1]. Although the surgical methods of laryngeal cancer are becoming more and more invasive and the treatment model is more and more accurate, there are still many patients with laryngeal cancer who have a poor quality of life after surgery. For laryngeal cancer patients, treatment is not only satisfied with complete removal of the tumor and reducing recurrence. And improve survival, while also gradually having greater expectations for the quality of life after surgery. A large number of studies have suggested that patients with laryngeal cancer have depression and anxiety after surgery, and that depression and anxiety affect the prognosis and quality of life of patients with laryngeal cancer $[2,3]$. In recent years, how to relieve postoperative depression and anxiety in patients with laryngeal cancer through nursing has been paid more and more attention $[4,5]$. It has been confirmed through clinical practice that the intervention of family members in the nursing mode intervention can effectively relieve the depression and anxiety of patients with laryngeal cancer after operation compared with conventional nursing. The report is as follows. 


\section{Materials and Methods}

\subsection{Clinical Data}

The subjects of this research were 40 patients with laryngeal cancer who were admitted from January 2017 to October 2019. They were randomly divided into two groups, including 20 patients with laryngeal cancer in the observation group, 18 males and 2 females, aged 34 to 82 years. The average age was $(65.24 \pm 2.25)$ years. Six cases underwent total laryngectomy, 11 cases underwent partial laryngectomy, and 3 cases underwent $\mathrm{CO}_{2}$ laser surgery. Postoperative pathological results showed that all patients had laryngeal squamous cell carcinoma. Both succeeded. The length of hospital stay was 14 to 32 days, with an average of (25.38 \pm 8.56) days. In the control group, there were 20 patients with laryngeal cancer, 17 males and 3 females, aged 35 to 81 years, with an average age of $(64.96 \pm 1.85)$ years, 5 of whom underwent total laryngectomy and 11 were performed with partial laryngectomy. Three patients underwent $\mathrm{CO}_{2}$ laser surgery. Postoperative pathological results showed that they were all larynx squamous cell carcinoma, and all of them were successful. The length of hospital stay was 15 to 30 days, with an average of $(21.52 \pm 4.55)$ days. There was no significant difference in age and length of hospital stay between the two groups, $P>0.05$. The research obtained the consent of the clinical department and the support of the Medical Ethics Association. The case selection was based on the voluntary principle, and the patients and their families signed informed consent. The subjects were agreed to participate in the psychological state assessment, and recorded the contact information of the patients and important relatives, such as telephone and WeChat for follow-up.

\subsection{Nursing Methods}

The two groups of patients were treated by the same group of doctors, methods of anesthesia and surgery were basically the same. Patients in the control group got routine laryngeal cancer nursing measures in our hospital. Patients were first evaluated after admission, and the patients were instructed to prepare blood before surgery, pre-/post-operation precautions, dietary guidance, etc.; Monitor vital signs, observe wound bleeding, tracheotomy care in trachea and fixation of outer tube, care of nasal feeding diet, rehabilitation training. On the basis of the control group, the observation group took family members to participate in nursing and jointly formulate a nursing plan. The entire nursing process was accompanied by the family members throughout the process. If the family members of patients are invited to participate in the patient care, to provide nursing assistance to the patients, to encourage the patients to actively face the disease with their affection, to overcome the disease. Let the patients feel the warmth and care from the family, and not be alone in the fight against the disease. Make the patient Generate a sense of security, reduce the negative psychology of patients, the care and companionship of family members or relatives and friends, so that patients can feel the care and existence of their loved ones, and give sufficient affirmation and encouragement to the patient's mental appearance and progress in treatment. The care and support of relatives, friends, and unit leaders help patients solve practical difficulties, give patients financial peace of mind, and reduce the pressure on patients. Provide health knowledge to patients' family about laryngeal cancer (relevant knowledge about hemi-laryngectomy, total laryngectomy, surgical methods, purpose, post-operative recovery time and related matters, etc.) by making health education before operation. Postoperative health knowledge (gastric tube, stool, indwelling urinary tube, diet, life, non-verbal communication, pronunciation training during recovery period) make up for the lack of family related knowledge of patients. For patients with long-term tube after total laryngeal surgery, explain the entire process of operation for patient's family members participate in the whole process of post-care until they have the ability to self-care. By nursing operation, they can master the correct nursing method for laryngeal cancer patients, which is better care. Instruct the family members to monitor the patient's condition, closely observe the patient's psychological and emotional conditions, notified the medical staff in time if any abnormalities are found. So the family members can supervise and urge the patient's postoperative rehabilitation, at the same time, encourage the patients to do it themselves and improve self-care ability.

\subsection{Observation Indicators}

The self-rating anxiety scale (SAS) and self-rating depression scale (SDS) compiled by Zung [6] were used to evaluate the anxiety and depression of patients. Both Chinese version of SAS and SDS have been proved to have good reliability and validity. SAS includes 20 items, with a total score of 50 to 59 points, 60 to 69 points, and 69 points are considered mild, moderate, and severe anxiety respectively; SDS includes 20 items, with a total score of 53 to 62 points, 63 to 72 points and 72 or higher are considered mild, moderate, or severe depression, respectively. The SAS and SDS scales were used to score anxiety and depression at both admission and discharge. The quality of life was assessed using the quality of life assessment scale in both groups $[6,7]$. The quality of life score consists of 5 items, each with 100 points. The higher the score, the better the quality of life. Nursing Satisfaction: Survey the patient's nursing satisfaction 2 hours before discharge. There are 8 answers in 8 aspects: satisfactory, relatively satisfactory, and unsatisfactory. 3 points, 2 points, and 1 point. Satisfied, 15 to 20 points are more satisfied, and 20 to 24 points are satisfied.

\subsection{Statistical Analysis}

SPSS15.0 statistical software was used for statistical analysis. The measurement data were analyzed by t-test and the count data were analyzed by $\chi^{2}$ test. $P<0.05$ was considered statistically significant.

\section{Results}

The SAS and SDS scores of the two groups were compared and compared. There was no significant difference in SAS and 
SDS scores between the two groups at admission $(P>0.05)$; the SAS and SDS scores of the two groups were significantly lower than those at admission $(P<0.05)$ The SAS score and SDS score of the observation group were significantly lower than those of the control group $(P<0.05)$. See Table 1 . The results of postoperative quality of life assessment and comparison between the two groups were significantly higher than those of the control group $(P<0.05)$. See Table 2 . Patients in the observation group also had higher nursing satisfaction than those in the control group. See Table 3.

Table 1. SAS and SDS score results and comparison between the two groups $(x \pm s)$.

\begin{tabular}{|c|c|c|c|c|c|}
\hline \multirow{2}{*}{ Group } & \multirow{2}{*}{ Cases } & \multicolumn{2}{|l|}{ SAS Score } & \multicolumn{2}{|l|}{ SDS Score } \\
\hline & & Admission & Discharge & Admission & Discharge \\
\hline Observation & 20 & $73.13 \pm 8.26$ & $46.62 \pm 7.52$ & $70.15 \pm 8.37$ & $41.53 \pm 5.24$ \\
\hline Control & 20 & $74.56 \pm 7.21$ & $63.12 \pm 10.14$ & $73.13 \pm 8.26$ & $58.35 \pm 5.19$ \\
\hline $\mathrm{t}$ value & & 6.13 & 4.35 & 6.09 & 4.11 \\
\hline $\mathrm{p}$ value & & $>0.05$ & $<0.05$ & $>0.05$ & $<0.05$ \\
\hline
\end{tabular}

Table 2. Evaluation results of postoperative quality of life between two groups $(x \pm s)$.

\begin{tabular}{llllll}
\hline Group & Cases & Physiology & Body & Emotion & Society \\
\hline Observation & 20 & $78.23 \pm 15.43$ & $79.45 \pm 16.34$ & $81.76 \pm 19.12$ & $78.76 \pm 4.12$ \\
Control & 20 & $62.76 \pm 10.24$ & $56.31 \pm 15.32$ & $71.44 \pm 12.54$ & $66.64 \pm 4.97$ \\
t value & & 6.46 & 6.35 & 6.90 & $80.12 \pm 3.87$ \\
p value & & $<0.05$ & $<0.05$ & $<0.05$ & $69.11 \pm 3.21$ \\
\hline
\end{tabular}

Table 3. Comparison of patient satisfaction between the two groups.

\begin{tabular}{|c|c|c|c|c|c|}
\hline Group & Cases & Satisfied & Quite satisfied & Not satisfied & Satisfaction (\%) \\
\hline Observation & 20 & 17 & 2 & 1 & $96 \%$ \\
\hline Control & 20 & 12 & 5 & 3 & $87 \%$ \\
\hline
\end{tabular}

Note: Comparison of satisfaction between the two groups, $\chi 2=3.24, \mathrm{P}<0.05$

\section{Discussion}

Depression and anxiety are common mental disorders that affect people's physical and mental health seriously. Anxiety refers to excessive worry and experience as a sign of restlessness, as if something terrible is going to happen. Depression is an emotional change in response to prolonged frustrated feelings caused by adverse external stimuli. It shows that patient is not interested in anything and their expression is indifferent. Most clinical depression and anxiety come from worrying about the disease itself. Laryngeal cancer is one of the common malignant tumors in otorhinolaryngology head and neck surgery. Currently the main treatment methods are surgery and radiotherapy [8]. After the operation, the patient's permanent tracheostomy and personal appearance changed, which induced the psychological burden of depression and anxiety. At the same time, postoperative airway mouth care and tumor recurrence further aggravated depression and anxiety, which seriously affected the patient's life. Quality and effect of disease treatment $[9,10]$. Some researches showed that, the incidence of postoperative anxiety was $23.9 \%$, and the incidence of depression was $33.7 \%$ [11]. Depression and anxiety are more common. Patient's age, education, surgical method, clinical stage, and marital status have become postoperative depression. The main factor of anxiety is to actively carry out targeted nursing interventions to alleviate depression and anxiety, reduce complications, and improve treatment effects. In recent years, the problem of depression and anxiety in patients with laryngeal cancer has been paid more and more attention [12]. The family participation nursing mode is a mode to guide family members to participate in the patient's detailed care. The patient's care process is accompanied by family members. The care mode makes the nursing staff more intensive and comprehensive, the patient care is more detailed for more easily accepted, and the nursing effect is better. It also lays the foundation for patients to enjoy continuous medical passports after discharge. Family participation in nursing can encourage patients' confidence to overcome the disease, and understand the patient's psychological state in a timely manner, provide psychological support, urge the patient's self-care activities, and improve the patient's self-care ability. The results of this study show that patients with laryngeal cancer have varying degrees of depression and anxiety after surgery, and their quality of life has deteriorated. However, patients' depression and anxiety can be effectively improved after family members participate in the nursing mode of nursing. At the same time, the quality of life and nursing satisfaction of patients in the observation group were significantly higher than those in the control group. It also clearly shows that by guiding the patient's family care measures to promote their participation in the prognostic care of patients with laryngeal cancer, while ensuring the treatment effect, it can also help to improve the patient's psychological state, improve patient self-care ability, and help prognosis.

In short, patients with laryngeal cancer can be provided with family participation mode nursing intervention on the basis of routine care after surgery. It have higher clinical significance, which is conducive to the improvement of patients' depression and anxiety, to improve the quality of postoperative rehabilitation, self-care ability, confidence, social support, the quality of life, and promote the recovery of 
patients' disease. However, the sample size of this study is small, and it is necessary to expand the sample for further research in the future to verify the conclusion and provide effective nursing methods to improve the clinical efficacy of laryngeal cancer patients after surgery.

\section{Acknowledgements}

The authors declare no conflict of interest.

\section{References}

[1] Bray F, Ferlay J, Soerjomataram I, et al. Global cancer statistics 2018: GLOBOCAN estimates of incidence and mortality worldwide for 36 cancers in 185 countries [J]. CA: A Cancer Journal for Clinicians, 2018.

[2] Devins GM, Payne AYM, Lebel S, et al. The burden of stress in head and neck cancer [J]. Psychooncology, 2013; 22 (3): 668-676.

[3] Tang PL, Wang C, Hung MF, et al. Assessment of Symptom Distress in Cancer Patients Before and After Radiotherapy [J]. Cancer Nursing; 34 (1): 78-84.

[4] Huang YH, Yang YL, Huang FC, et al. MicroRNA-29a mitigation of endoplasmic reticulum and autophagy aberrance counteracts in obstructive jaundice-induced fibrosis in mice $[\mathrm{J}]$. Exp Biol Med (Maywood), 2018; 243 (1): 13-21.

[5] Joseph LA, Routledge JA, Burns MP, et al. Value of the Hospital Anxiety and Depression Scale in the follow up of head and neck cancer patients [J]. Journal of Laryngology \& Otology, 2013; 127 (3): 1-10.
[6] Baregamian N, Song J, Bailey CE, et al. Tumor necrosis factor-alpha and apoptosis signal-regulating kinase 1 control reactive oxygen species release, mitochondrial autophagy, and c-Jun N-terminal kinase/p38 phosphorylation during necrotizing enterocolitis [J]. Oxid Med Cell Longev, 2009; 2 (5): 297-306.

[7] Maringwa JT, Quinten C, King M, et al. Minimal important differences for interpreting health-related quality of life scores from the EORTC QLQ-C30 in lung cancer patients participating in randomized controlled trials [J]. 19 (11): 1753-1760.

[8] Amorin BDS, Maria RM, Aparecido DR, et al. Quality of life and depression in patients undergoing total and partial laryngectomy [J]. Clinics; 60 (2).

[9] Vahratian A, Smith YR, Dorman M, et al. Longitudinal depressive symptoms and state anxiety among women using assisted reproductive technology [J]. 95 (3): 0-1194.

[10] Chen AM, Daly ME, Vazquez E, et al. Depression Among Long-term Survivors of Head and Neck Cancer Treated With Radiation Therapy [J]. JAMA Otolaryngology-Head \& Neck Surgery; 139 (9): 885.

[11] Yilmaz M, Yener M, Yollu U, et al. Depression, self esteem and sexual function in laryngeal cancer patients $[\mathrm{J}]$. Clinical otolaryngology: official journal of ENT-UK; official journal of Netherlands Society for Oto-Rhino-Laryngology \& Cervico-Facial Surgery, 2015; 40 (4): 349.

[12] Bergstrom L, Ward EC, Finizia C. Voice rehabilitation after laryngeal cancer: Associated effects on psychological well-being [J]. Supportive Care in Cancer. 